\title{
Meningitis bacteriana aguda por Streptococcus agalactiae en una mujer no embarazada asociada a una fístula de LCR: comunicación de un caso
}

\author{
Bernardita González, Tomás Labatut, Andrés Soto, Alberto Fica y Marcelo Castro
}

\section{Acute bacterial meningitis by Streptococcus agalactiae in a non pregnant woman associated to a cerebrospinal fluid leak: a case report}

Streptococcus agalactiae is a rare cause of acute bacterial meningitis. We report the case of a middle age nonpregnant female patient, with no comorbitidies, who was hospitalized with acute meningitis. The pathogen was identified both in blood and CSF. She recovered uneventfully with ceftriaxone and dexamethasone. A CSF leak was suspected by previous history of unilateral watery rhinorrhea, that was demonstrated with a high resolution paranasal sinus CT and beta-2 transferrin analysis of the nasal fluid. Vulvovaginitis was also diagnosed after admission, but no cultures were obtained. Streptococcus agalactiae is an infrequent cause of bacterial meningitis that should promote the search of anatomical abnormalities or comorbidities in non-pregnant adults and beyond newborn period.

Key words: Bacterial meningitis, Streptococcus agalactiae, cerebrospinal fluid leak.

Palabras clave: Meningitis bacteriana, Streptococcus agalactiae, fístula de líquido cefalorraquídeo.

\section{Introducción}

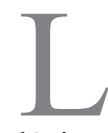

a meningitis bacteriana aguda (MBA) es una de las infecciones más frecuentes del sistema nervioso central (SNC) en adultos, siendo los agentes etiológicos más frecuentes Streptococcus pneumoniae y Neisseria meningitidis ${ }^{1}$. Sin embargo, en algunas ocasiones puede ser causada por otros microorganismos, tales como Mycobacterium tuberculosis, Listeria monocytogenes y Treponema pallidum.

Streptococcus agalactiae es una bacteria que, si bien suele estar asociada a infecciones genitourinarias y con mayor frecuencia en mujeres embarazadas, ha sido descrito como causa infrecuente de meningitis en adultos ${ }^{2,3}$.

Se presenta el primer caso reportado en Chile de meningitis por S. agalactiae en una mujer adulta inmunocompetente, no embarazada, con una alteración anatómica como probable factor predisponente.

\section{Caso clínico}

Paciente de sexo femenino, 52 años, con antecedentes de un TEC simple hace 20 años sin secuelas y con un cuadro de rinorrea acuosa de tres semanas de evolución, sin fiebre ni cefalea. Consultó en otorrinolaringología donde se le diagnosticó una probable rinitis vasomotora. Se prescribió corticoides intranasales y sistémicos (prednisona $10 \mathrm{mg}$ al día) y azitromicina por siete días.
Luego de una mejoría transitoria del cuadro presentó una cefalea holocránea de inicio súbito, asociado a un dolor cervical, compromiso de conciencia sin focalidad neurológica evidente y calofríos, por lo que consultó en el Servicio de Urgencia de nuestro hospital. Al ingreso destacaban cifras tensionales límites (presión arterial $108 / 70 \mathrm{~mm} \mathrm{Hg})$ que respondieron a volumen, fiebre $\left(\mathrm{T}^{\circ}\right.$ $38,5^{\circ} \mathrm{C}$ ), desorientación temporo-espacial, agitación psicomotora y signos meníngeos. Se tomaron hemocultivos y se efectuó una punción lumbar que dio salida a LCR turbio, citoquímico: 8.480 céls $/ \mathrm{mm}^{3}, 100 \%$ polimorfonucleares, proteínas $370 \mathrm{mg} / \mathrm{dl}$ y glucosa $2 \mathrm{mg} / \mathrm{dl}$. Con el diagnóstico de una MBA se inició tratamiento empírico con ceftriaxona $2 \mathrm{~g}$, dos veces al día y dexametasona i.v. $10 \mathrm{mg}, 4$ veces al día, esta última por 4 días. La tomografía axial computada (TAC) de cerebro no mostró alteraciones significativas. Fue admitida en la Unidad de Pacientes Críticos, donde evolucionó sin requerimiento de fármacos vasoactivos, con caída de la curva febril y recuperación en su condición neurológica en menos de $24 \mathrm{~h}$.

En la tinción de Gram del LCR no se observaron bacterias y la tinción naranja de acridina mostró formas cocoides. Tanto en el LCR como en los hemocultivos hubo identificación de $S$. agalactiae susceptible a penicilina, eritromicina y clindamicina. El laboratorio de referencia (Instituto de Salud Pública de Chile) confirmó posteriormente los hallazgos de los cultivos, informando además que correspondía al serotipo Ib.

La paciente fue evaluada por ginecología encontrándo-
Hospital Militar de Santiago, Chile.

Departamento de Medicina, Servicio de Infectología (BG, AS, AF).

Servicio de Otorrrinolaringología (TL).

Servicio de Imagenología (MC)

Recibido: 8 de mayo de 2013 Aceptado: 5 de agosto de 2013

Correspondencia a: Alberto Fica Cubillos albertofica@gmail.com 
se la presencia de una vulvovaginitis y miomas uterinos, no se realizó estudio microbiológico. Al examen otorrinolaringológico se observó una rinorrea acuosa abundante en la fosa nasal izquierda evidenciada con la maniobra de Valsalva. Por la sospecha de una fístula de LCR se realizó una TAC de cavidades paranasales de alta resolución que reveló pérdida de continuidad a nivel de la lámina cribosa del etmoides izquierdo y adelgazamiento de la pared postero-lateral del hemiseno esfenoidal izquierdo en su aspecto más craneal con engrosamiento mucoso (Figura $1)$. Se solicitó un estudio de $\beta 2$-transferrina del líquido nasal, que resultó positivo. Se realizó además una TAC de abdomen y pelvis que no reveló hallazgos de importancia.

Su evolución clínica fue favorable, con disminución progresiva de los parámetros inflamatorios, completando 14 días con ceftriaxona. Se decidió el alta con una programación diferida de resolución quirúrgica de la fístula de LCR. Se recomendó la vacunación antineumocócica polisacárida y antimeningocócica cuadrivalente conjugada.

En forma dirigida la paciente refirió golpes menores recurrentes en la cabeza varias semanas previas al ingreso con un muro debajo de una escalera que le permitía acceder a un patio común para tender ropa. La paciente reconoció además haber ocupado el mismo papel higiénico para limpiarse la zona genital y nasal unos días antes del ingreso por la escasez de éste en el baño, durante la visita a unos familiares y atendiendo a su intensa rinorrea.

Tras el alta la paciente no volvió a presentar salida de LCR por la nariz. A pesar de esto, debido a que presentaba una fístula de probable origen espontáneo y que ya había tenido un episodio de MBA, se le aconsejó someterse a una cirugía endoscópica nasal para reparar la zona dehiscente en la base del cráneo anterior.

\section{Discusión}

Streptococcus agalactiae es una bacteria cocácea grampositiva, capsulada, anaerobia facultativa, $\beta$ hemolítica, catalasa y oxidasa negativa. Dentro de su características, presenta el antígeno polisacárido B de Lancefield y otros antígenos proteicos que permiten su clasificación en 10 serotipos diferentes ( $\mathrm{Ia}, \mathrm{Ib}, \mathrm{II}-\mathrm{IX}$ ), siendo los serotipos III, Ia y II los más frecuentes en Chile ${ }^{4}$.

Streptococcus agalactiae pertenece a la microbiota normal del tracto digestivo humano y puede también colonizar el tracto genital, rectal, urinario y rara vez la nasofaringe. En algunas ocasiones puede producir invasión a la sangre y al SNC. Es causa importante de bacteriemia y meningitis en neonatos, con una tasa de incidencia anual de 0,6 a 1,7 casos por 1.000 recién na$\operatorname{cidos}^{4}$; sin embargo, actualmente también es reconocido como agente responsable de infecciones en adultos ${ }^{3}$.

Si bien se ha descrito un aumento en la incidencia de

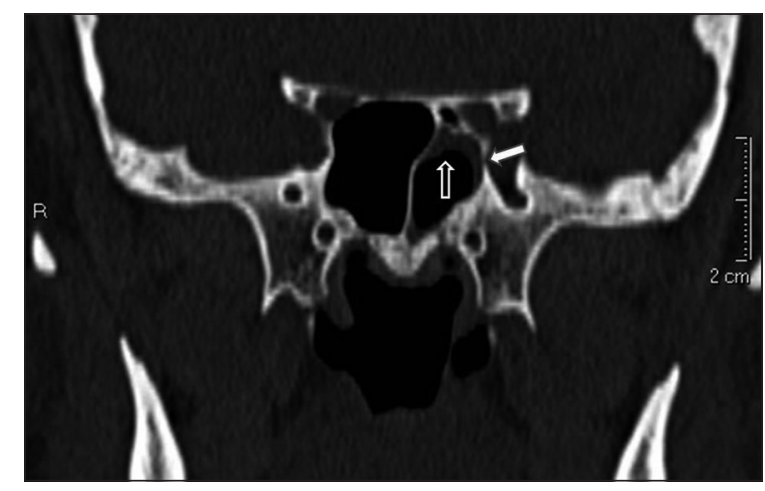

Figura 1. Tomografía computada de alta resolución de cavidades paranasales en proyección coronal. Se aprecia defecto en la pared lateral del seno esfenoidal izquierdo (flecha blanca) y una reacción mucoperióstica adyacente (flecha negra de contorno blanco).

casos de enfermedades invasoras provocadas por este microorganismo en las últimas décadas ${ }^{2,3,5}$, la MBA continúa siendo una manifestación infrecuente. De hecho, no hay evidencias de casos similares descritos previamente en pacientes adultos en Chile y sólo se conoce un caso publicado en Latinoamérica (Argentina) ${ }^{6}$.

Clásicamente se ha descrito la enfermedad invasora por $S$. agalactiae en relación al embarazo; sin embargo, las medidas de prevención de la enfermedad neonatal han permitido que actualmente más de dos tercios de los casos de patología invasora por este agente en los E.U.A se manifiesten en adultos, sobre todo en aquellos con co-morbilidades tales como diabetes mellitus (20-25\% de los casos), cirrosis hepática, cáncer de mama y vejiga neurogénica, entre otros ${ }^{2}$.

El espectro clínico de la infección por $S$. agalactiae en los adultos puede ser numeroso y variable, desde infecciones de piel y tejidos blandos, hasta osteomielitis, endocarditis, neumonía y meningitis ${ }^{2,3}$.

Se ha descrito que $S$. agalactiae sería responsable de $4 \%$ de los casos de las MBA en adultos; la mayoría de ellos en mujeres en el post-parto, adultos mayores o con co-morbilidades significativas ${ }^{1,2}$. Con menor frecuencia se asocia a procedimientos neuroquirúrgicos o fístulas de LCR ${ }^{1}$. Los síntomas suelen ser agudos, en general indistinguibles de los otros agentes causales de MBA y se asocia a bacteriemia hasta en $80 \%$ de los casos. Con frecuencia suele encontrarse un foco de infección distal, en el endometrio o endocardio.

La tasa de mortalidad de la MBA por $S$. agalactiae llega a $34 \%^{1}$ y se postula que contribuyen a un peor pronóstico la edad avanzada y la presencia de complicaciones neurológicas o extra-neurológicas; pese a ello, se ha descrito que la gran mayoría de los pacientes que sobreviven a esta condición presentan una recuperación completa $^{1,7}$. 
Una fístula de LCR es una comunicación anormal entre dos cavidades que da salida a este líquido fuera de sus trayectos habituales. La génesis de este tipo de anormalidades anatómicas requiere de la pérdida de continuidad entre la barrera ósea y duramadre que rodea al hueso, produciendo cefalea, rinorraquia u otorraquia según el sitio de la fístula. Sin embargo, la principal importancia de una fístula de LCR radica en que constituye una potencial puerta de entrada para distintos microorganismos al SNC, siendo responsable de hasta $20 \%$ de los casos de $\mathrm{MBA}^{8}$. La etiología es diversa, siendo la traumática la causa más frecuente presente en hasta $50 \%$ de los casos ${ }^{9}$. Otras etiologías son la iatrogénica, malformaciones congénitas, hidrocéfalo, erosiones óseas espontáneas y tumores de la silla turca. El diagnóstico puede ser confirmado, con una alta sensibilidad y especificidad, mediante la utilización de métodos de laboratorio, como la inmunoelectroforesis de $\beta 2$-transferrina, una proteína que se encuentra presente en el LCR y ausente en la sangre y secreciones nasales.

El estudio radiológico debe hacerse mediante una TAC de alta resolución para identificar el defecto óseo, la cual debe complementarse con una resonancia magnética (RM) para descartar posibles meningoencefaloceles asociados. Una cisterno-TAC con medio de contraste intratecal puede ayudar a una mejor localización de la fístula aunque el hecho de que esta prueba de un resultado negativo no descarta su presencia ${ }^{10}$.

El manejo de las fístulas de LCR nasales le compete al otorrinolaringólogo e involucra un equipo multidisciplinario integrado por infectólogos, neurólogos y neurocirujanos según las necesidades de cada caso en particular. Este puede ser conservador o quirúrgico.

El tratamiento conservador consiste en 7 a 10 días de reposo en cama con la cabecera elevada a $30^{\circ}$, evitar esfuerzos, tos y estornudos, así como también hacer uso de laxantes, todas medidas encaminadas a evitar el aumento de la presión intracraneal. Está indicado en el manejo inicial de aquellas fistulas de origen traumático o iatrogénico. Se puede recurrir al uso de un drenaje lumbar continuo de 5-10 $\mathrm{ml}$ de LCR por hora lo cual requiere un estricto monitoreo y manipulación por personal de enfermería especializado ${ }^{11}$. No está claro el uso de antimicrobianos sistémicos como profilaxis aunque sí se recomienda su uso en casos post-cirugía endoscópica nasal debido a la contaminación previa del sitio operatorio ${ }^{12}$. Los diuréticos como la acetazolamida pueden ser un adyuvante terapéutico útil en el caso de fístulas espontáneas con presión intracraneal elevada ${ }^{13}$.

El riesgo de desarrollar una MBA existiendo una fístula de LCR es de aproximadamente $10 \%$ por año ${ }^{10}$. Este riesgo existe aún a pesar de no tener rinolicuorrea activa ${ }^{14}$. Es por eso que la cirugía de las fístulas de LCR nasal está indicada cuando fracasa el tratamiento conservador (traumáticas y iatrogénicas) o en las de origen espontáneo.
Respecto a la causa de la fístula de la paciente descrita en el caso clínico, el origen espontáneo es el más probable por el antecedente de rinorrea clara de comienzo insidioso y la localización del defecto óseo objetivado en la TAC. Los traumatismos leves repetidos, como los reportados por la paciente, no son el tipo de trauma que habitualmente produce fístulas ya que cuando es éste el origen, normalmente existe el antecedente de un TEC de alta energía. El abordaje quirúrgico utilizado para la reparación del defecto ha sufrido un vuelco importante con el desarrollo de la cirugía endoscópica nasal ${ }^{15}$. La técnica endoscópica es hoy el tratamiento de elección gracias a su excelente visualización del defecto, baja morbilidad y tasas de más de $90 \%$ de éxito ${ }^{16-18}$. La técnica quirúrgica consiste en la colocación de un injerto que cubre la zona dehiscente que comunica la fosa craneal anterior con la fosa nasal. Para esto se utiliza preferentemente tejido del propio paciente como hueso, cartílago, colgajos de mucoperiostio, grasa o fascia así como también pegamentos biosintéticos ${ }^{19}$.

Es importante destacar que las fístulas de origen espontáneo requieren especial atención ya que puede existir un posible cuadro de hipertensión intracraneal subyacente como factor causal. Cuando una fistula espontánea se presenta en mujeres de edad media y sobrepeso, este factor etiológico se debe descartar mediante una punción lumbar con medición de presión intracraneal. Si se confirma un alza en la presión intracraneal hay que considerar el uso de un drenaje lumbar en el post-operatorio así como el uso de diuréticos ya que la tasa de fracaso en el cierre quirúrgico es más alta en estos $\operatorname{casos}^{20}$.

Varios factores concurren en el caso aquí presentado y su rol específico es difícil de asignar. Por una parte tenemos la aparición de una fístula de LCR, el uso reciente de corticoides sistémicos y tópicos, una vulvovaginitis y una posible colonización nasal desde la microbiota vaginal. Lamentablemente no se contó con un estudio microbiológico vaginal para demostrar esta hipótesis.

En conclusión, la aparición de un cuadro de MBA por $S$. agalactiae fuera del contexto de un embarazo o en el período peri-parto, obliga a buscar co-morbilidades o defectos anatómicos en el cráneo. Este último fenómeno puede ser sospechado ante un trauma mayor o como en este caso por traumas aparentemente menores y el desarrollo de una rinorrea acuosa abundante unilateral.

\section{Resumen}

Streptococcus agalactiae es una causa infrecuente de meningitis bacteriana aguda. Comunicamos el caso de una mujer de edad media sin co-morbilidades que ingresó por un cuadro de meningitis producido por este patógeno, el que también fue identificado en hemocultivos. La paciente se trató con ceftriaxona y corticoesteroides i.v., 
recuperándose satisfactoriamente. Por el antecedente de una rinorrea acuosa unilateral, se sospechó una fístula de LCR, la que se demostró con una tomografía computada multicorte de senos paranasales y por una prueba de $\beta 2$ transferrina en fluido nasal. En forma concomitante se diagnosticó una vulvovaginitis sin estudio microbiológico. Streptococcus agalactiae es una causa infrecuente de meningitis bacteriana que si no se presenta asociada al embarazo o en neonatos, obliga a la búsqueda de anormalidades anatómicas o co-morbilidades.

\section{Referencias bibliográficas}

1.- Domingo P, Barquet N, Álvarez M, Coll P, Nava J, Garau J. Group B streptococcal meningitis in adults: Report of twelve cases and review. Clin Infect Dis 1997; 25: 1180-7.

2.- Farley M M. Group B Streptococcal disease in nonpregnant adults. Clin Infect Dis 2001; 33 : 556-61.

3.- Sarmiento R, Wilson F M, Khatib R. Group B streptococcal meningitis in adults: case report and review of the literature. Scand J Infect Dis 1993; 25: 1-6.

4.- Instituto de Salud Pública de Chile. Vigilancia de laboratorio enfermedad invasora Streptococcus agalactiae. Boletín Instituto de Salud Pública de Chile 2012; 2 (10). Disponible en: http://www.ispch.cl/sites/default/files/ boletin_streptococcus_agalactiae_n10.pdf. Fecha acceso: 8 de mayo de 2013.

5.- Martins E R, Florindo C, Martins F, Aldir I, Borrego M J, Brum L, et al. Streptococcus agalactiae serotype $\mathrm{Ib}$ as an agent of meningitis in two adult nonpregnant women. J Clin Microbiol 2007; 45: 3850-2.

6.- Roel J E, Pí AO, Pescio A, Bouza G, Boero A, Gonzales-Moles D. Meningitis aguda por Streptococcus agalactiae en una paciente adulta con fistula de líquido cefalorraquídeo. Medicina (B. Aires) 1997; 57: 64-6.
7.- Chotmongkol V, Poonsriaram A. Streptococcus agalactiae meningitis in adults: report of two cases. J Med Assoc Thai 2002; 85: 385-7.

8.- Bachmann-Harildstad G. Diagnostic values of beta- 2 transferrin and beta-trace protein as markers for cerebrospinal fluid fistula. Rhinology 2008; 46: 82-5.

9.- Schmidt T, Rebolledo V, Kawaguchi K, Santamaría A, Pinto J. Abordaje endoscópico de las fístulas de líquido cefalorraquídeo. Rev Otorrinolaringol Cir Cabeza Cuello 2003; 63: 112-6.

10.- Jones N S, Becker D G. Advances in the management of CSF leaks. BMJ 2001; 322: 122-3.

11.- Meco C, Oberascher G. Comprehensive algorithm for skull base dural lesion and cerebrospinal fluid fistula diagnosis. Laryngoscope 2004; 114: 991-9.

12.- Hegazy H M, Carrau R L, Snyderman C H, Kassam A, Zweig J. Transnasal endoscopic repair of cerebrospinal fluid rhinorrhea: a metaanalysis. Laryngoscope 2000; 110: 1166-72.

13.- Mirza S, Thaper A, McClelland L, Jones N S. Sinonasal cerebrospinal fluid leaks: management of 97 patients over 10 years. Laryngoscope 2005; 115: 1774-7.

14.- Bernal-Sprekelsen M, Alobid I, Mullol J, Trobat F, Tomás-Barberán M. Closure of cerebrospinal fluid leaks prevents ascending bacterial meningitis. Rhinology 2005; 43 : 277-81.

15.- Kerr J T, Chu F W, Bayles S W. Cerebrospinal fluid rhinorrhea diagnosis and management. Otolaryngol Clin North Am 2005; 38 : 597- 611.

16.- Zweig J L, Carrau R L, Celin S E, Schaitkin B M, Pollice P A, Snyderman C H, et al. Endoscopic repair of cerebrospinal fluid leaks to the sinonasal tract: predictors of success. Otolaryngol Head Neck Surg 2000; 23: 195-201.

17.- Lindstrom D R, Toohill R J, Loehrl T A, Smith T L. Management of cerebrospinal fluid rhinorrhea: the Medical College of Wisconsin experience. Laryngoscope 2004; 114: 969-74.

18.- Briggs R J, Wormald P J. Endoscopic transnasal intradural repair of anterior skull base cerebrospinal fluid fistulae. J Clin Neurosci 2004; 11: 597-9.

19.- Cassano M, Felippu A. Endoscopic treatment of cerebrospinal fluid leaks with the use of lower turbinate grafts: a retrospective review of 125 cases. Rhinology 2009; 47: 362-8.

20.- Wise S K, Schlosser R J. Evaluation of spontaneous nasal cerebrospinal fluid leaks. Curr Opin Otolaryngol Head Neck Surg 2007; 15: 28-34. 